\title{
Covid-19 Breakthrough Infections in Vaccinated People. A Clinical-Epidemiological Case Series Reporting of 30 Patients in Toledo (Spain) from February to September 2021
}

\section{Turabian JL*}

Specialist in Family and Community Medicine, Regional Health Service of Castilla la Mancha (SESCAM), Spain

*Corresponding author: Jose Luis Turabian, Health Center Santa Maria de Benquerencia, Regional Health Service of Castilla la Mancha (SESCAM), Toledo, Spain, Email: jturabianf@ hotmail.com

\section{Research Article}

Volume 5 Issue 4

Received Date: October 23, 2021

Published Date: November 26, 2021 DOI: $10.23880 /$ eij-16000208

\section{Abstract}

Background: COVID-19 vaccines show excellent efficacy, but some people still become infected with SARS-CoV-2 after vaccination.

Objective: To study, in general medicine, COVID-19 breakthrough infections in vaccinated people

Methodology: An observational, longitudinal and prospective case series study of COVID-19 breakthrough infections in vaccinated people, based on a prospective cohort of patients was carried out from February 1, 2021 to September 30, 2021, in a general medicine office in Toledo (Spain)

Results: During the 8 months of the study, 30 patients with COVID-19 breakthrough infections in vaccinated people were included, with a mean age of 65 years. 53\% were women. 73\% were symptomatic. Mild symptoms of ENT predominated (33\%), followed by respiratory (27\%). 57\% had chronic diseases. $60 \%$ of the cases had been vaccinated with BNT162b2 mRNA vaccine (Comirnaty, Pfizer/BioNTech) and 20\% with ChAdOx1 nCoV-19 vaccine (Vaxzevria, Oxford / AstraZeneca).

Conclusion: In the context of general medicine in Toledo (Spain), during the first 8 months of COVID-19 vaccination, COVID-19 breakthrough infections in vaccinated people occur in older adults with chronic diseases, without gender differences, and frequently they are mild symptomatic. Each of the vaccines has associated breakthrough infections.

Keywords: COVID-19; SARS-CoV-2; Vaccination; Breakthrough Infection; General Practice; Case Series

Abbreviations: WHO: World Health Organization; PCR: Polymerase Chain Reaction.

\section{Introduction}

On December 31, 2019, the World Health Organization (WHO) reported a series of cases of pneumonia caused by an unknown agent in the city of Wuhan, China. In January 2020 , it was reported that the cause of this infection was a new coronavirus called SARS-CoV-2 and the disease caused by it, COVID-19. The global repercussions of the epidemic were quickly evident, which is why in March 2020 the WHO granted it the category of pandemic [1]. As of October 4, 2021 COVID-19 has caused more than 234 million confirmed cases and 4 million deaths $[2,3]$.

An unprecedented feat has occurred in this situation: developing and administering a vaccine against the new 


\section{Epidemiology International Journal}

pathogen in just 338 days after the genetic sequence was performed [4]. Since December 2020, several vaccines have been licensed by regulators such as the European Medicines Agency, the US Food and Drug Administration, and the UK Medicines and Health Products Regulatory Agency [5-9]. Currently, the European Commission has licensed four vaccines: BNT162b2 mRNA vaccine (Comirnaty, Pfizer/ BioNTech), licensed December 21, 2020; mRNA-1273 vaccine Spikevax (formerly COVID-19 Moderna vaccine, licensed January 6; ChAdOx1 nCoV-19 vaccine (AstraZeneca vaccine), licensed 29 December and the Janssen/Johnson \& Johnson vaccine, authorized on March 11. In Spain, these four vaccines are currently available, all of which have been approved by the European Medicines Agency [10]. At present, including all the vaccines developed, more than 6,000 million vaccines have been administered $[2,3]$.

Data presented in different studies and other phase 3 clinical trials have demonstrated a robust efficacy of these vaccines $(>85 \%)$ in preventing severe symptomatic disease [11-17]. But some people still become infected with SARS-CoV-2 after vaccination. More than a quarter of fully vaccinated patients admitted to hospital with SARSCoV-2 have been reported to be seriously or critically ill with COVID-19; This could be a reflection of numerous factors, including the appearance of SARS-CoV-2 variants that could confer a decreased efficacy of the vaccine, an ineffective immune response among people with comorbidities (eg, older age, overweight, and use immunosuppressive agents, as well as those living in more disadvantaged areas), and the ability to prevent asymptomatic infection [18-27].

Spain began its mass immunization program on December 27, 2020, shortly after the first COVID-19 vaccine (BNT162b2 mRNA vaccine; Comirnaty, Pfizer/BioNTech) was approved earlier that month. With data of September 30, 2021, in Castilla La Mancha, (Spain), 85\% of the population has a complete COVID-19 vaccination schedule [28]. Breakthrough cases should be investigated to assess how COVID-19 vaccines work in real-world conditions. This is important for [29]:

- Learning if vaccines offer the same protection seen in clinical trials.

- Adjusting vaccine recommendations, as needed.

- Learning why and how often breakthrough cases (people getting sick after the two-week period after their final dose) occur.

- Learning how vaccines protect against COVID-19 variants.

In this context, we present an observational, longitudinal and prospective study in general medicine in Toledo, Castilla La Mancha (Spain), based on a cohort of patients from February 1,2021 to September 30,2021, which aimed to know and describe the clinical-epidemiological characteristics of patients who consult for COVID-19 breakthrough infections.

\section{Material and Methods}

\section{Design and Emplacement}

An observational, longitudinal and prospective case series study of COVID-19 breakthrough infections in vaccinated people, based on a prospective cohort of patients was carried out from February 15, 2021 to September 31, 2021, in a general medicine office in the Health Center Santa Maria de Benquerencia, Toledo (Castilla La Mancha, Spain), which has a list of 2,000 patients $>14$ years of age (in Spain, the general practitioners [GPs] care for people $>14$ years of age, except for exceptions requested by the child's family and accepted by the GP). The GPs in Spain work within the National Health System, which is public in nature, and are the gateway for all patients to the system, and each person is assigned a GP [30].

\section{Outcome of Interest}

The outcome of interest was characterizing COVID-19 breakthrough infections in vaccinated people.

\section{Diagnosis of COVID-19 Breakthrough Infections in Vaccinated People}

Because the vaccines require about two weeks reaching their maximum effectiveness, a person is not considered fully vaccinated until two weeks after they completed the recommended number of doses for the vaccine they received. Therefore, for public health surveillance purposes, a case of COVID-19 vaccine breakthrough is defined as someone who tests positive (PCR or antigen) for COVID-19 being fully vaccinated.

To consider a person as fully vaccinated, it was required:

- That they have received 2 doses of vaccine separated by a minimum of 19 days if the first dose was BNT162b2 mRNA vaccine (Comirnaty, Pfizer/BioNTech), 21 days in the case of ChAdOx1 nCoV-19 vaccine (Vaxzevria, Oxford/AstraZeneca) or 25 days in the case of mRNA1273 vaccine (Spikevax, formerly COVID-19 Vaccine Moderna), and that a minimum period of 7 days has elapsed since the last dose if the last dose was with BNT162b2 mRNA vaccine (Comirnaty), or 14 days if it was with ChAdOx1 $\mathrm{nCoV}-19$ vaccine (Vaxzevria) or mRNA-1273 vaccine (Spikevax). People who received a dose of Janssen vaccine (Johnson \& Johnson vaccine) more than 14 days ago were also considered fully vaccinated.

- Or, that having passed the disease they have received a 


\section{Epidemiology International Journal}

dose of any of the vaccines, after the minimum period equal to that established for the second doses.

- In the heterologous regimen in which Vaxzevria (Oxford/ AstraZeneca) is used in the first dose and mRNA vaccines in the second, it was considered fully vaccinated after 7 days if the second dose was with Comirnaty or after 14 days if it was with the Moderna vaccine [31].

\section{COVID-19 Diagnosis}

Diagnosis was performed with reverse transcriptase polymerase chain reaction (PCR) oropharyngeal swab test or antigen test for symptomatic patients with less than 5 days of evolution [32]. The PCR tests were performed both in symptomatic patients and in asymptomatic contacts. A symptomatic confirmed case with active infection was considered to be any person with a clinical picture of sudden onset acute respiratory infection of any severity that occurs, among others, with fever, cough or feeling of shortness of breath. Other symptoms such as odynophagia, anosmia, ageusia, musc le pain, diarrhea, chest pain or headache, among others, were also considered symptoms of suspected SARS-CoV-2 infection according to clinical criteria; and a positive PCR or rapid antigen test positive [33].

\section{Collected Variables}

Data were extracted from the medical records of the general medicine practice under study. The following variables were collected:

- Age and sex.

- Symptoms of COVID-19 breakthrough infections in vaccinated people.

- Chronic diseases (defined as "any alteration or deviation from normal that has one or more of the following characteristics: is permanent, leaves residual impairment, is caused by a non-reversible pathological alteration, requires special training of the patient for rehabilitation, and/or can be expected to require a long period of control, observation or treatment" [34,35], classified according to the International Statistical Classification of Diseases and Health-Related Problems, CD-10 Version: 2019.

- Duration of symptoms in days of COVID-19 breakthrough infections in vaccinated people.

- Social-occupancy class de COVID-19 breakthrough infections in vaccinated people (according to the Registrar General's classification of occupations and social status code) [36,37].

- If they were Health Care Workers.

- Complex family and low income household based on the genogram and in the experience of the general practitioner about continuity of care and knowledge of the family (genogram was a schematic model of the structure and processes of a family, which included the family structure, life cycle and family relational patterns. It was understood that "complex" genogram identified complex families with psychosocial problems) [38-41].

- Ethnic minority.

- $\quad$ Sick leave due to COVID-19 breakthrough infections in vaccinated people.

- Symptomatic/asymptomatic COVID-19 breakthrough infections in vaccinated people.

- Severity of COVID-19 breakthrough infections in vaccinated people (mild cases: clinical symptoms are mild and no manifestation of pneumonia could not be found on images; moderate cases: with symptoms such as fever and respiratory tract symptoms, and the manifestation of pneumonia can be seen on the imaging tests; and severe cases: respiratory distress, respiratory rate $\geq 30$ breaths/min., pulse oxygen saturation $\leq 93 \%$ with room air at rest, arterial partial pressure of oxygen/ oxygen concentration $\leq 300 \mathrm{mmHg}$.) [42]; to simplify comparison, moderate and severe cases were counted together.

- Vaccine type: BNT162b2 mRNA vaccine (Comirnaty, Pfizer/BioNTech), mRNA-1273 vaccine (Spikevax, formerly COVID-19 Vaccine Moderna), ChAdOx1 nCoV19 vaccine (Vaxzevria, Oxford/AstraZeneca), and Janssen (Johnson \& Johnson vaccine).

\section{Sample}

All patients who consulted for COVID-19 breakthrough infections in vaccinated people from February 1, 2021 to September 3o, 2021 were included, and that they were seen in the consultation object of the study and their medical documentation was available.

\section{Results}

Thirty patients with COVID-19 breakthrough infections in vaccinated people (CBIVP) were included, with a mean age of $65.5+-14.71$ (Range: $26-84$ years), $27 \%$ were $>=65$ years, and $23 \%>=45$ years old. $53 \%$ were women. Only 1 patient (3\%) had had a previous COVID-19. 73\% were symptomatic. The duration of symptoms in days of the COVID-19 breakthrough infections in vaccinated people was $5.63+-4.00$ (Range: 2-20 days) (Tables 1-4). ENT symptoms predominated (33\%: Anosmia 1, rhinorrhea 3, odynophagia 11 , pharyngeal mucus 3 , nasal congestion 5), followed by respiratory (27\%: chest pain 1, cough 18$)$. There were 2 severe infections (one pneumonia, and another patient with pneumonia and ovarian vein thrombosis). $17 \%$ were health workers. 33\% required sick leave. 13\% were ethnic minority. $57 \%$ had chronic diseases: musculo-skeletal (24\%), endocrine (16\%), Digestive system (15\%), and Nervous and Senses and Circulatory system (13\%) were predominated. 


\section{Epidemiology International Journal}

$60 \%$ of the cases had been vaccinated with BNT162b2 mRNA vaccine (Comirnaty, Pfizer/BioNTech) and 20\% with ChAdOx1 nCoV-19 vaccine (Vaxzevria, Oxford/AstraZeneca).
There were no patients vaccinated with the heterologous regimen.

\begin{tabular}{|c|c|}
\hline Variables & $\begin{array}{c}\text { Covid-19 Breakthrough Infections In } \\
\text { Vaccinated People } \mathrm{N}=30 \\
\end{array}$ \\
\hline Age in years (Arithmetic mean +- Standard deviation; Range) & 65.5+-14.71 (Range: $26-84$ years) \\
\hline$>=65$ years & $8(27)$ \\
\hline$=<45$ years & $7(23)$ \\
\hline$=<18$ years & 0 \\
\hline Women & $16(53)$ \\
\hline Previous symptomatic COVID-19 (and one dose of vaccine) & $1(3)$ \\
\hline Previous asymptomatic COVID-19 (and one dose of vaccine) & 0 \\
\hline Previous COVID-19 with moderate-severe severity & 0 \\
\hline Symptomatic COVID-19 in breakthrough infections in vaccinated people & $22(73)$ \\
\hline $\begin{array}{c}\text { Duration of symptoms in days of COVID-19 in breakthrough infections in vaccinated } \\
\text { people(Arithmetic mean + - Standard deviation; Range) }\end{array}$ & $5.63+-4.00$ (Range: $2-20$ days) \\
\hline $\begin{array}{l}\text { COVID-19 breakthrough infections in vaccinated people with severity moderate and } \\
\text { severe }\end{array}$ & $\begin{array}{c}2 \text { (7) [2 pneumonia and } 1 \text { ovarian vein } \\
\text { thrombosis] }\end{array}$ \\
\hline Social-occupancy class of patients (people with some type of labour specialization) & $10(33)$ \\
\hline Health Care Workers with COVID-19 breakthrough infections in vaccinated people & $5(17)$ \\
\hline Sick leave for COVID-19 breakthrough infections in vaccinated people & $10(33)$ \\
\hline Ethnic minority with COVID-19 breakthrough infections in vaccinated people & $4(13)$ \\
\hline Low income household in COVID-19 breakthrough infections in vaccinated people & $4(13)$ \\
\hline Complex family with COVID-19 breakthrough infections in vaccinated people & $1(3)$ \\
\hline $\begin{array}{l}\text { Chronic diseases presence in COVID-19 breakthrough infections in vaccinated } \\
\text { people }\end{array}$ & $17(57)$ \\
\hline
\end{tabular}

(): Denotes percentages

Table 1: Frequency of Selected Variables in the Case Series of Covid-19 Breakthrough Infections in Vaccinated People.

\begin{tabular}{|c|c|}
\hline Symptoms * According To Who, Icd-10 Groups & $\begin{array}{c}\text { Covid-19 Breakthrough Infections In Vaccinated } \\
\text { People N=30 }\end{array}$ \\
\hline General (discomfort, asthenia, myalgia, fever, arthralgias) & 15 (21) [Fever 10, asthenia 2, discomfort 3] \\
\hline Respiratory (cough, dyspnea, chest pain) & 19 (27) [chest pain 1, cough 18] \\
\hline $\begin{array}{c}\text { ENT (anosmia/ageusia, odynophagia, rhinorrhea, pharyngeal } \\
\text { dryness-mucus, epixtasis) }\end{array}$ & $\begin{array}{c}23 \text { (33) [Anosmia 1, rhinorrhea 3, odynophagia 11, } \\
\text { pharyngeal mucus 3, nasal congestion 5] }\end{array}$ \\
\hline Digestive (anorexia, nausea/vomiting, diarrhea, abdominal pain) & 5 (7) [Anorexia 2, vomiting, 1, diarrhea 2] \\
\hline Neurological (headache, dizziness, mental confusion -brain fog) & 8 (12) [Headache 6, dizziness 2] \\
\hline Psychiatric (anxiety, insomnia) & 0 \\
\hline Skin (chilblains, flictenas, rash) & 0 \\
\hline Total symptoms* & 70 (100) \\
\hline
\end{tabular}

(): Denotes percentages

* Patients could have more than one symptom. The percentages are over the total of symptoms

Table 2: Symptoms in Covid-19 Breakthrough Infections in Vaccinated People. 


\begin{tabular}{|c|c|}
\hline Chronic Diseases* According To Who, Icd-10 Groups & Covid-19 Breakthrough Infections In Vaccinated People N=30 \\
\hline -III Diseases of the blood & $2(2)$ \\
\hline -IV Endocrine & $13(16)$ \\
\hline -V Mental & $4(5)$ \\
\hline -VI-VIII Nervous and Senses & $10(13)$ \\
\hline -IX Circulatory system & $10(13)$ \\
\hline -X Respiratory system & $3(4)$ \\
\hline -XI Digestive system & $12(15)$ \\
\hline -XII Diseases of the skin & 0 \\
\hline -XIII Musculo-skeletal & $19(24)$ \\
\hline -XIV Genitourinary & $6(8)$ \\
\hline TOTAL chronic diseases* & $79(100)$ \\
\hline
\end{tabular}

(): Denotes percentages

* Patients could have more than one chronic disease. The percentages are over the total of chronic diseases

Table 3: Chronic Diseases in Covid-19 Breakthrough Infections in Vaccinated People.

\begin{tabular}{|c|c|}
\hline Vaccine Type & $\begin{array}{l}\text { Covid-19 Breakthrough Infections In Vaccinated } \\
\text { People } \mathrm{N}=30\end{array}$ \\
\hline BNT162b2 mRNA vaccine (Comirnaty, Pfizer/BioNTech) & $18(60)$ \\
\hline $\begin{array}{c}\text { mRNA-1273 vaccine (Spikevax, formerly COVID-19 Vaccine } \\
\text { Moderna) }\end{array}$ & $1(3)$ \\
\hline ChAdOx1 nCoV-19 vaccine (Vaxzevria, Oxford/AstraZeneca) & $6(20)$ \\
\hline Janssen (Johnson \& Johnson vaccine) & $5(17)$ \\
\hline
\end{tabular}

( ): Denotes percentages

Table 4: Vaccine Type in Covid-19 Breakthrough Infections in Vaccinated People.

\section{Discussion}

Since the start of the vaccination campaign, the development of COVID-19 has been reported in people who have received one or both doses of the vaccine [43]. In public health, a "breakthrough case" is when a fully vaccinated person later gets the disease they were vaccinated for. No vaccine provides $100 \%$ protection against infection, so breakthrough cases are not new, and not unique, to COVID-19. People fully vaccinated against COVID-19 are less likely to become infected with the coronavirus if they have relatively high levels of virus-blocking antibodies, according to a study of thousands of healthcare workers who received BNT162b2 mRNA vaccine (Comirnaty, Pfizer/BioNTech). Analysis of the data on the ChAdOx1 nCoV-19 vaccine (Vaxzevria, Oxford/ AstraZeneca) also showed a link between higher levels of neutralizing antibodies and a lower likelihood of progressive infection [44]. It has been considered that with respect to the three pillars of the immune system (antibodies, B cells and T cells), vaccination stimulated long-lasting cellular immunity; memory B cells continued to grow in number for at least six months and improved at fighting the virus over time; $\mathrm{T}$ cell counts were relatively stable, declining only slightly over time [45].

\section{Severity and Hospitalization}

Although the trend of COVID-19 breakthrough infections in vaccinated people is worrying, they remain relatively rare, and those that cause serious illness, hospitalization or death even more. More than 97 percent of people hospitalized for COVID-19 are not vaccinated. Still, vaccinated people can get asymptomatic or mild infections. The dose is also important: a vaccinated person exposed to a low dose of the coronavirus may never become infected, or not be noticeably infected [46]. Likewise, the vaccine has been shown to reduce the incidence of asymptomatic infection and associated infectivity [12,47-53]. Our study repeats these data: COVID-19 breakthrough infections in vaccinated people were rare: 29 cases in 8 months for a study population of 2000 people, and with an official complete vaccination rate of $85 \%$ [28]; and predominantly with mild symptoms $(67 \%$; 20 of 30 ) or asymptomatic (27\%; 8 of 30 ); only 2 cases were hospitalized. 


\section{Epidemiology International Journal}

The benefits of these vaccines are substantial and their rapid implementation is an important public health achievement [54], although protection after two doses of BNT162b2 mRNA vaccine (Comirnaty, Pfizer/BioNTech) decreased from $88 \%$ in one month to $74 \%$, between five and six months after vaccination; and the protection of the ChAdOx1 nCoV-19 vaccine (Vaxzevria, Oxford/AstraZeneca) decreased from $77 \%$ at one month to $67 \%$ at four to five months [55]. Four out of 10 people who are clinically vulnerable generate lower levels of antibodies than healthy recipients after two injections of the SARS-CoV-2 vaccine, according to a study that included patients with solid organ and hematologic cancers, end-stage kidney and liver disease, organ transplants, and immune-mediated inflammatory disease, such as inflammatory bowel disease, vasculitis, or rheumatoid arthritis [56].

\section{Symptoms}

Research shows that in vaccinated people the presence of the virus is confined to the nose and nasopharynx, while the lungs are free In our study, generally mild symptoms of ENT (33\%: Anosmia 1, rhinorrhea 3, odynophagia 11, pharyngeal mucus 3 , nasal congestion 5) were predominant [57]. We also found a short duration of symptoms (mean of $4.7+-2.2$ days, in the 20 symptomatic patients not hospitalized), reducing the risk of prolonged COVID-19. On the other hand, it has been reported that the symptoms of COVID-19 change for those vaccinated: Fever was no longer the most permanent symptom, loss of taste and smell either, and rhinorrhea, sore throat or dry cough and headache appeared as very frequent symptoms in people with the vaccine [58]. Our results share this finding.

\section{Comorbidities}

Thanks to the vaccine, there are now only three reasons why there may be people admitted, and that highly reduces the risk: In the first place, a vaccinated person could become infected again if his defenses have not responded well to the vaccines; secondly if, after several months, it has lost potency, something that can happen in people with a delicate immune system; and lastly, in people who have risk factors such as diabetes, obesity or asthma. Certain comorbidities, including heart disease and lung disease, have been reported to be significantly associated with post vaccination infection. We found that $57 \%$ had chronic diseases, predominantly musculo-skeletal (24\%), endocrine (16\%), digestive system $(15 \%)$, and nervous and senses and circulatory system (13\%).

\section{Health Workers}

The risk of reporting a positive SARS-CoV-2 test is higher among frontline healthcare workers than the general population probably reflecting greater exposure and testing. We found that $17 \%$ were health workers.

\section{Age and Gender}

As of April 2021, the population infected with vaccination in the United States consisted of $63 \%$ women (proportional to the proportion of vaccinated); the median age was 58 years [59]. Our data indicate that there was no difference in COVID-19 breakthrough infections in vaccinated people by sex, and that they predominated in older adults with a mean age of $65.5+-14.71(27 \%$ in $>=65$ years $)$.

\section{Vaccines}

Each of the vaccines has associated breakthrough infections. The majority of COVID-19 breakthrough infections in vaccinated people in our series of cases were vaccinated with BNT162b2 mRNA vaccine (Comirnaty, Pfizer/BioNTech), which simply translates the fact that the majority of the population was vaccinated with that vaccine. In the absence of denominators, these data do not allow conclusions to be drawn.

\section{Limitations of the Study}

Our study has several limitations:

- First, although we provided extensive documentation of a cohort of COVID-19 breakthrough infections in vaccinated people, the number of cases was relatively small.

- Second, they may have missed asymptomatic cases that did not attend in GP consultation, as no surveillance or systematic screening was done.

\section{Conclusion}

COVID-19 breakthrough infections in vaccinated people occur in older adults with chronic diseases; without gender differences, and are frequently mild symptomatic, with ENT and respiratory symptoms predominant. Most were vaccinated with Pfizer-BioNTech-BNT162b2 (Pfizer/ BioNTech) mRNA, which simply translates the fact that the majority of the population was vaccinated with that vaccine. Our data show that each of the vaccines has associated breakthrough infections, and with these small numbers we cannot report that one is more or less effective in our population. Our findings could support the advice to be prudent in relaxing physical distancing and other personal protection measures in the post-vaccination era, particularly around frail older adults, even if these people are vaccinated, and this could have implications for strategies such as booster vaccines. 


\section{References}

1. Ayarza IS, Bachelet VC (2021) What we know and don't know on SARS-CoV-2 and COVID-19. Medwave 21(4): e8198.

2. (2020) Coronavirus COVID-19 Global Cases by the Center for Systems Science and Engineering (CSSE) at Johns Hopkins. The Center for Systems Science and Engineering (CSSE) at JHU.

3. (2021) WHO coronavirus disease (COVID-19) dashboard. World Health Organization.

4. Heaton PM (2021) Herd Immunity: The Journey Is as Important as the Destination. J Infect Dis 224(6): 931933.

5. Polack FP, Thomas SJ, Kitchin N, Absalon J, Gurtman A, et al. (2020) Safety and efficacy of the BNT162b2 mRNA Covid-19 vaccine. N Engl J Med 383: 2603-2615.

6. Voysey M, Clemens SAC, Madhi SA, Weckx LY, Folegatti PM, et al. (2021) Safety and efficacy of the ChAdOx1 nCoV-19 vaccine (AZD1222) against SARS-CoV-2: an interim analysis of four randomized controlled trials in Brazil, South Africa, and the UK. Lancet 397(10269): 99111.

7. Baden LR, El Sahly HM, Essink B, Kotloff K, Frey S, et al. (2021) Efficacy and safety of the mRNA-1273 SARSCoV-2 vaccine. N Engl J Med 384(5): 403-416.

8. Logunov DY, Dolzhikova IV, Shcheblyakov DV, Tukhvatulin AI, Zubkova OV, et al. (2021) Safety and efficacy of an rAd26 and rAd5 vector-based heterologous prime-boost COVID-19 vaccine: an interim analysis of a randomised controlled phase 3 trial in Russia. Lancet 397(10275): 671-681.

9. Tanne JH (2020) Covid-19: FDA panel votes to approve Pfizer BioNTech vaccine. BMJ 371: m4799.

10. (2021) Vaccination campaign against COVID-19. Ministry of Health.

11. Dagan N, Barda N, Kepten E, Miron O, Perchik S, et al. (2021) BNT162b2 mRNA Covid-19 vaccine in a nationwide mass vaccination setting. N Engl J Med 384: 1412-1123.

12. Vasileiou E, Simpson CR, Robertson C, Shi T, Kerr S, et al. (2021) Effectiveness of first dose of COVID-19 vaccines against hospital admissions in Scotland: national prospective cohort study of 5.4 million people. Preprint with the Lancet.

\section{Epidemiology International Journal}

13. Thompson MG, Burgess JL, Naleway AL, Tyner Al, Yoon SK, et al. (2021) Interim estimates of vaccine effectiveness of BNT162b2 and mRNA-1273 COVID-19 vaccines in preventing SARS-CoV-2 infection among health care personnel, first responders, and other essential and frontline workers-eight U.S. locations, December 2020-March 2021. MMWR Morb Mortal Wkly Rep 70(13): 495-500.

14. Daniel W, Nivet M, Warner J, Podolsky DK (2021) Early evidence of the effect of SARS-CoV-2 vaccine at one medical center. N Engl J Med 384: 1962-1963.

15. Britton A, Jacobs Slifka KM, Edens C, Nanduri SA, Bart SM, et al. (2021) Effectiveness of the Pfizer-BioNTech COVID-19 vaccine among residents of two skilled nursing facilities experiencing COVID-19 outbreaks-Connecticut, December 2020-February 2021. MMWR Morb Mortal Wkly Rep 70(11): 396-401.

16. Janssen Biotech (2021) Janssen Ad26.COV2.S vaccine for the prevention of COVID-19. FDA briefing document, pp: $1-62$.

17. Cabezas C, Coma E, Fernandez NM, Li X, Marcos MM, et al. (2021) Associations of BNT162b2 vaccination with SARS-CoV-2 infection and hospital admission and death with covid-19 in nursing homes and healthcare workers in Catalonia: prospective cohort study. BMJ 374: n1868.

18. Juthani PV, Gupta A, Borges KA, Price CC, Lee AI, et al. (2021) Hospitalization among vaccine breakthrough COVID-19 infections. Lancet Infect Dis 21(11): 14851486.

19. Glatter RD, Antevy PM, Rasmussen AM, Iovine NM (2021) COVID Positive after Two Vaccine Doses? What It Means. Medscape.

20. Amit S, Beni SA, Biber A, Grinberg A, Leshem E, et al. (2021) Post-vaccination COVID-19 among healthcare workers, Israel. Emerg Infect Dis 27(4): 1220-1222.

21. Wadman M (2021) The overlooked superpower of mRNA vaccines. Science 373(6554): 479.

22. Antonelli M, Penfold RS, Merino J, Sudre J, Molteni E, et al. (2021) Risk factors and disease profile of postvaccination SARS-CoV-2 infection in UK users of the COVID Symptom Study app: a prospective, communitybased, nested, case-control study. Lancet Infect Dis, pp: 1-13.

23. Leshem E, Nelson K, Lopman BA (2021) Severe breakthrough COVID-19 infections in Scotlandimplications for immunisation programmes. Lancet 
Infect Dis, pp: 1-2.

24. Burioni R, Topol EJ (2021) Has SARS-CoV-2 reached peak fitness?. Nat Med 27: 1323-1324.

25. (2021) The father of the Oxford vaccine sees group immunity with the Delta variant as impossible. El Mundo.

26. Butt AA, Hego HN, Chemaitelly H, Samra ABA, Khal AA, et al. (2021) Outcomes among Patients with Breakthrough SARS-CoV-2 Infection After Vaccination. Int J Infect Dis 110: 353-358.

27. Wells BW (2021) My Family Was a COVID Cluster. Four vaccinated adults and two unvaccinated children had mild symptoms. Is this what the end of the pandemic looks like?. The New Yorker.

28. (2021) Percentage of the vaccinable population by age group that has a complete dose and schedule. Sanidad Castilla La Mancha.

29. (2021) Breakthrough cases: Tracking disease infection after vaccination. South Carolina Department of Health and Environmental Control.

30. Turabian JL (1995) Notebooks of Family and Community Medicine. An introduction to the principles of Family Medicine. Madrid: Díaz de Santos.

31. (2021) COVID-19 early detection, surveillance and control strategy. Ministerio de Sanidad, pp: 1-32.

32. Liu W, Russell RM, Ruche FB, Skelly AN, Mix SS, et al. (2021) Predictors of Nonseroconversion after SARSCoV-2 Infection. Emerg Infect Dis 27(9): 2454-2458.

33. (2020) Strategy for early detection, surveillance and control of COVID-19. Instituto de Salud Carlos III, pp: 1-32.

34. Strauss AL (1984) Chronic illness and the quality of life. St Louis: The C.V. Mosby Company.

35. WHO (2020) International Statistical Classification of Diseases and Health-Related Problems. ICD-10 Version: 2019.

36. (1986) The Classification and Analisis of General Practice Data. Royal Collage of General Practitioners.

37. Donaldson RJ, Donaldson LJ (1983) Essential Community Medicine. Lancaster: MTP Press.

38. Turabian JL (2017) Family Genogram in General Medicine: A Soft Technology that can be Strong. An Update. Res Med Eng Sci 3(1): 186-191.

\section{Epidemiology International Journal}

39. Russell LT (2020) Capturing Family Complexity in Family Nursing Research and Practice. J Fam Nurs 26(4): 287293.

40. Watts C, Shrader E (1998) How to do (or not to do)... The genogram: a new research tool to document patterns of decision-making, conflict and vulnerability within households. Health Policy Plan 13(4): 459-464.

41. Mcllvain H, Crabtree B, Medder J, Stange KC, Miller WL (1998) Using practice genograms to understand and describe practice configurations. Fam Med 30(7): 490496.

42. Mao S, Huang T, Yuan H, Li M, Huang X, et al. (2020) Epidemiological analysis of 67 local COVID-19 clusters in Sichuan Province, China. BMC Public Health 20: 1525.

43. Keehner J, Horton LE, Pfeffer MA, Longhurst CA, Currier JS, et al. (2021) SARS-CoV-2 Infection after Vaccination in Health Care Workers in California: N Engl J Med 384: 1774-1775.

44. Mallapaty S (2021) A blood marker predicts who gets 'breakthrough' COVID. Real-world evidence from a medical centre links high levels of potent antibodies after vaccination to a reduced risk of infection. Nature.

45. Dolgin E (2021) COVID vaccine immunity is waning-how much does that matter? As debates about booster shots heat up, what's known about the duration of vaccinebased immunity is still evolving. Nature, pp: 1-2.

46. Mandavilli A (2021) Why Vaccinated People Are Getting 'Breakthrough' Infections. The vaccines are effective at preventing serious illness and death, but they are not a golden shield against the coronavirus. The New York Times.

47. Yochay GR, Amit S, Bergwerk M, Lipsitch M, Leshem E, et al. (2021) Decreased infectivity following BNT162b2 vaccination: a prospective cohort study in Israel. Lancet Reg Health Eur 7: 100150.

48. Petter E, Mor O, Zuckerman N, Levi DO, Younger A, et al. (2021) Initial real world evidence for lower viral load of individuals who have been vaccinated by BNT162b2. MedRxiv, pp: 1-9.

49. Bernal JL, Andrews N, Gower C, Robertson C, Stowe J, et al. (2021) Effectiveness of the Pfizer-BioNTech and Oxford-AstraZeneca vaccines on covid-19 related symptoms, hospital admissions, and mortality in older adults in England: Test negative case-control study. BMJ 373: n1088.

50. Bergwerk M, Gonen T, Lustig Y, Amit S, Lipsitch M, et al. 
(2021) Covid-19 Breakthrough Infections in Vaccinated Health Care Workers. N Engl J Med 385: 1474-1484.

51. Steenhuysen J (2021) COVID-19 Vaccines Hold Strong Against Delta, Protection Waning in Older Adults: Medscape.

52. Self WH, Tenforde MW, Rhoads JP, Gaglani M, Ginde AA, et al. (2021) Comparative Effectiveness of Moderna, PfizerBioNTech, and Janssen (Johnson \& Johnson) Vaccines in Preventing COVID-19 Hospitalizations Among Adults Without Immunocompromising Conditions-United States, March-August 2021. MMWR Morb Mortal Wkly Rep 70(38): 1337-1343.

53. Haas EJ, Angulo FJ, McLaughlin JM, Anis E, Singer SR, et al. (2021) Impact and effectiveness of mRNA BNT162b2 vaccine against SARS-CoV-2 infections and COVID-19 cases, hospitalisations, and deaths following a nationwide vaccination campaign in Israel: an observational study using national surveillance data. Lancet 397(10287): 1819-1829.

54. Dean N (2021) Hospital admissions due to COVID-19 in

\section{Epidemiology International Journal}

Scotland after one dose of vaccine. Lancet 397(10285): 1601-1603.

55. Iacobucci G (2021) Covid-19: Protection from two doses of vaccine wanes within six months, data suggest. BMJ 374: n2113.

56. Munro C (2021) Covid-19: 40\% of patients with weakened immune system mount lower response to vaccines. BMJ 374: n2098.

57. Cuppini L (2021) What happens when you get Covid being vaccinated?. El Mundo.

58. Mejía L (2021) Immunologists warn: "We have to maximize precautions until there are no more vaccinated". La Ventana.

59. (2021) COVID-19 Vaccine Breakthrough Case Investigations Team. COVID-19 vaccine breakthrough infections reported to CDC-United States, January 1-April 30, 2021. MMWR Morb Mortal Wkly Rep 70(21): 792-793. 